\title{
BMJ Open Competency of health workers in detecting and managing gestational hypertension, pre-eclampsia, severe pre- eclampsia and eclampsia during antenatal check-ups in primary care health facilities in Bangladesh: a cross- sectional study
}

\author{
Sk Masum Billah, ${ }^{1,2}$ Abdullah Nurus Salam Khan (10 , , ${ }^{1,3}$ S M Rokonuzzaman, \\ Nafisa Lira Huq, ${ }^{1}$ Marufa Aziz Khan, ${ }^{4}$ Sabrina Sharmin Priyanka, ${ }^{1}$ \\ Imteaz Ibne Mannan, ${ }^{5}$ Setara Rahman, ${ }^{6}$ Shams El Arifeen, ${ }^{1}$ Joby George ${ }^{7}$
}

To cite: Billah SM, Khan ANS, Rokonuzzaman SM, et al. Competency of health workers in detecting and managing gestational hypertension, pre-eclampsia, severe preeclampsia and eclampsia during antenatal check-ups in primary care health facilities in Bangladesh: a crosssectional study. BMJ Open 2021;11:e046638. doi:10.1136/ bmjopen-2020-046638

- Prepublication history and additional supplemental material for this paper are available online. To view these files, please visit the journal online (http://dx.doi.org/10.1136/ bmjopen-2020-046638).

Received 05 November 2020 Accepted 06 July 2021

Check for updates

(c) Author(s) (or their employer(s)) 2021. Re-use permitted under CC BY-NC. No commercial re-use. See rights and permissions. Published by BMJ.

For numbered affiliations see end of article.

Correspondence to

Sk Masum Billah;

billah@icddrb.org

\section{ABSTRACT}

Study objective To evaluate the competency of trained health workers in detecting and managing hypertensive disorders of pregnancy during routine antenatal check-ups (ANCs) at primary care facilities in Bangladesh.

Study design and settings Cross-sectional study; conducted in 26 primary care facilities.

Outcome measures Accurate diagnosis of the hypertensive disorders of pregnancy.

Method In total 1560 ANC consultations provided by primary health workers, known as Family Welfare Visitors (FWVs), were observed using a structured checklist between October 2017 and February 2018. All consultations were reassessed by study physicians for validation.

Result Of the 'true' cases of gestational hypertension $(n=32)$, pre-eclampsia $(n=29)$ and severe pre-eclampsia $(n=16)$, only $3 \%, 7 \%$ and $25 \%$, respectively, were correctly diagnosed by FWVs. Per cent agreement for the diagnosed cases of any hypertensive disorders of pregnancy was $9 \%$ and kappa statistics was 0.50 (p value 0.0125 ). For identification of any hypertensive disorders by FWVs, sensitivity and positive predictive values were $14 \%$ and $50 \%$, respectively. There was a moderate positive correlation between the blood pressure measurements taken by FWVs and study physicians. Only $27 \%$ of those who had 'some protein' in urine were correctly identified by FWVs. Women diagnosed with any of the hypertensive disorders of pregnancy by FWVs were more likely to be counselled on at least one danger sign of pre-eclampsia (severe headache, blurring of vision and upper abdominal pain) than those without any such diagnosis ( $41 \%$ vs $19 \%$, $p$ value 0.008 ). All four cases of severe pre-eclampsia diagnosed by FWVs were given a loading dose of intramuscular magnesium sulphate and three among them were referred to a higher facility.

Conclusion The FWVs should be appropriately trained on risk assessment of pregnant women with particular
Strengths and limitations of this study

The study used a direct-observation based approach to evaluate the competency of health workers in diagnosing and managing hypertensive disorders of pregnancy during antenatal check-ups and also validated the accuracy of the performed activities (blood pressure (BP) measurement and urinalysis).

- Post-hoc analysis based on the two diagnostic criteria, elevated BP and protein in the urine as per the national guideline, indicates the percentage of 'true' cases of hypertensive disorders of pregnancy accurately diagnosed to be markedly increased.

- The study assessed the competency of health workers in primary care facilities of rural areas in Bangladesh, therefore, the findings are not generalisable to health workers working in the higher level of health facilities.

- The study could not determine the extent to which the deviations of measurements from the 'true' values were attributed to the error of the instruments (BP machine or urine test kits) since the instruments used by the health workers were not validated.

- Hawthorn effect was possible by the presence of observers and study physicians, however, their continual presence over 2 weeks in the selected facilities could potentially minimise the effect.

emphasis on accurately assessing the diagnostic criteria of hypertensive disorders of pregnancy and its management.

\section{INTRODUCTION}

Pre-eclampsia, a multisystem hypertensive disorder of pregnancy, is associated with high blood pressure (BP; diastolic BP >90 mm Hg) 
and the presence of protein in urine. ${ }^{1}$ The risks of adverse maternal and perinatal health outcomes are high when an advanced form of pre-eclampsia develops and leads to episodes of convulsion-a condition known as eclampsia. Eclampsia may lead to serious maternal health consequences including renal failure ${ }^{2}$ and placental abruption ${ }^{3}$ and accounts for $10 \%$ of maternal deaths worldwide. ${ }^{45}$ Despite the substantial reduction of maternal mortality ratio over the past decade in Bangladesh, ${ }^{6}$ eclampsia remains the second major direct cause of obstetric deaths constituting about $20 \%$ of all maternal mortalities. ${ }^{7}$ The condition is also associated with adverse fetal outcomes such as stillbirth, preterm delivery and intrauterine growth restriction. ${ }^{8}$

The key strategy to mitigate the burden related to preeclampsia is to diagnose the condition early so that timely and appropriate management can prevent further complications. ${ }^{9}$ The provision of injectable magnesium sulphate as the first stage management of severe pre-eclampsia can prevent the severity of its progression and lowers the risk of convulsion. ${ }^{10}$ The availability of life-saving interventions alone, however, does not ensure its delivery to the beneficiaries without improving the overall service provision for maternity care by skilled health workers. ${ }^{11}$ Some studies reported poor quality of antenatal check-up (ANC) regarding the detection and management of pre-eclampsia and observed incomplete physical examinations by the ANC providers. ${ }^{12} 13$ Lack of accuracy in BP measurement and urinary protein assessment can further compromise the detection of hypertensive disorders, including pre-eclampsia during pregnancy. There is, however, a scarcity of evidence on the accuracy of the clinical examinations conducted by the ANC providers.

In Bangladesh, female primary level health workers, known as Family Welfare Visitors (FWVs) with 18 months of preservice training, provide ANC services at community level primary healthcare centres (known as union health and family welfare centre or UH\&FWC) and community outreach centres (known as satellite clinics). ${ }^{14}$ The FWVs are designated to identify, manage and refer pre-eclampsia cases, and provide initial management of severe pre-eclampsia before referring the pregnant women to health facilities for comprehensive emergency obstetric care (CEmOC).${ }^{15}$ It is, therefore, crucial to accurately perform the essential components of ANC by the FWVs for accurate screening, diagnosis and subsequent management of pre-eclampsia or severe pre-eclampsia. ${ }^{16}$ The essential components of ANC include taking an appropriate history, measuring BP, assessing urinary protein and applying the appropriate clinical guidelines. ${ }^{17}$

The study objective is to evaluate the competency of FWVs in diagnosing and managing hypertensive disorders of pregnancy including gestational hypertension, pre-eclampsia, severe pre-eclampsia and eclampsia. The study also validated the $\mathrm{BP}$ measurement and urinary protein assessment conducted by FWVs by comparing the values with those assessed by study physicians (gold standard). This study was conducted during the provision of routine antenatal services by the FWVs at the UH\&FWCs and satellite clinics in Bangladesh.

The study will identify the quality gaps and the missed opportunities for the detection and management of hypertensive disorders in pregnancy during the ANC in primary care settings in Bangladesh. The findings will guide the maternal health programmers and policymakers to design effective interventions for improving the accuracy of antenatal screening for hypertensive disorders of pregnancy and reinforce the implementation of the national screening and management protocols for pre-eclampsia and eclampsia.

\section{METHODOLOGY}

\section{Study design}

We conducted a cross-sectional study at UH\&FWCs and satellite clinics, two important primary healthcare service delivery platforms for mothers and children in Bangladesh. UH\&FWCs are situated at the lowest administrative units (union) of the country and governed by the Directorate General of Family Planning of Ministry of Health and Family Welfare in Bangladesh and serve a population of 24000-30 000 on average. ${ }^{14}$ In each UH\&WFC, one female health worker, known as 'FWV', is posted to provide antenatal, childbirth and postnatal care, and family planning services 6 days a week. In addition to the regular UH\&FWC-based services, FWVs conduct outreach sessions that is, attend satellite clinics on two selected days every week. These sessions are usually held in a preidentified household of the village, a school premise, or a community clinic so that FWVs can provide the standard antenatal care and family planning services close to the community. The FWVs carry the required logistics for antenatal care to the satellite clinics, such as registers, job aids and counselling materials, BP machines with the stethoscope, urine test kits, weighing machine and essential medicines to provide standard ANC services.

Methods of data collection included-(i) observation of ANC services provided by FWVs by trained paramedics and (ii) reassessment of the pregnant women by trained physicians which was considered the gold standard for validating the FWV's assessment.

\section{Study site}

We conducted the study in 26 UH\&FWCs selected purposively from four subdistricts of three districts (Habiganj, Noakhali and Lakshmipur) in Bangladesh. In these three districts, The United States Agency for International Development (USAID) funded MaMoni Health Systems Strengthening (MaMoni HSS) project provided technical support to effectively implement the management of hypertensive disorders of pregnancy through existing government health workers. ${ }^{18}$ The project trained the FWVs in the selected UH\&FWCs on detection, prevention and management of hypertensive disorders of pregnancy using the loading dose of intramuscular magnesium sulphate according to the standard protocol between 
March and May of 2016. The 2-day long training was conducted by expert consultants following the CEmOC training manual developed by the Obstetric and Gynaecological Society of Bangladesh. MaMoni HSS also ensured the availability of injectable magnesium sulphate and 'urine strip' for urine assessment in government health facilities including UH\&FWC, subdistrict and district hospitals through a strong liaison with the government in these districts. The project also introduced referral slips to ensure that the identified severe pre-eclampsia or eclampsia cases at the UH\&FWCs could be referred to the designated CEmOC health facilities of the respective districts for further management. The project also provided infrastructural and skill improvement inputs to improve the quality of CEmOC services at the referral facilities. MaMoni HSS's inputs at different levels for implementing appropriate detection and management of pre-eclampsia and eclampsia at antenatal care contact points of the public facilities have been described elsewhere in detail. ${ }^{18}$

\section{Data collection}

We recruited six female paramedics with 4 years of training by government authorised 'Medical Assistant Training School' and three physicians for the study. After recruitment, the paramedics and the physicians received a 3-day training on ANC and hypertensive disorders of pregnancy using the national protocol for detection and management of pre-eclampsia and eclampsia. Following the training, both study paramedics and physicians participated in a demonstration on the usage of BP machine and urine assessment kit for standardisation in the steps of assessment and recording the result. Before starting the actual data collection, the assessors spent 2 days in a government training hospital for practising the assessment tools, BP machine and urine test kits.

Study paramedics stayed in the ANC consultation room and collected observation-based data when the FWV provided the ANC services. They observed ANC service provided by the FWVs both at UH\&FWCs and satellite clinics using a structured observation checklist. The checklist was developed from the components of the WHO focused ANC guideline and incorporated the detail of steps for diagnosing and managing hypertensive disorders of pregnancy. ${ }^{17} 19$ The observers recorded whether the health worker asked about the relevant history of current and previous pregnancy (if any), performed routine examinations such as measuring $\mathrm{BP}$ and assessing urine for protein and made a diagnosis for any of the four hypertensive disorders of pregnancy-gestational hypertension, pre-eclampsia, severe pre-eclampsia and eclampsia. The observers also recorded the diastolic and systolic BP, and the findings from urine assessment as measured by the FWVs in the checklist. If the FWVs provided the first dose (ie, the loading dose) of injectable magnesium sulphate to a diagnosed case of severe pre-eclampsia and eclampsia as per the protocol and/ or referred the pregnant woman to CEmOC equipped health facilities, it was also recorded in the checklist. They also extracted the obstetric and background information of the pregnant women from the FWV's ANC registers.

After completion of ANC service provision by the FWV, gold-standard study physicians reassessed every pregnant woman for the symptoms of hypertensive disorders of pregnancy following the standard national protocol. The study physicians measured the diastolic and systolic BP and tested urine for the presence of protein and recorded the information in a structured form. Study physicians conducted the reassessments in a space with auditory and visual privacy and separate from the routine ANC consultation room so that the reassessment activities were less likely to bias the routine practices of FWVs. The physicians independently applied the national protocol to identify the presence of any of the four conditions of hypertensive disorders. To avoid bias during the reassessment of a woman, the study physicians were unaware of any diagnosis or management provided by the FWVs before the reassessment. For example, where an FWV managed a diagnosed case of pre-eclampsia or severe pre-eclampsia by administering the first dose of injectable magnesium sulphate, the study physicians still reassessed the woman. In another situation, where an FWV missed the diagnosis of hypertensive disorders of pregnancy but the case was identified by the study physician, on ethical grounds, the woman was sent back to the FWV with a suggestion from the study physician to re-evaluate the woman. According to the ethical principles, once these women were diagnosed with hypertensive disorders of pregnancy by physicians (gold-standard) and were referred to a re-evaluation by FWV, it was guaranteed that these women received appropriate management and followed the instructions given by the FWV.

Between October 2017 and February 2018, we conducted observation and reassessment of a total of 1560 ANC consultations, 955 in UH\&FWCs and 605 in satellite clinics. The study physicians also assessed the readiness status of the 26 selected UH\&FWCs for screening and managing hypertensive disorders of pregnancy using a structured health facility assessment checklist. The checklist included information on maternal service availability, human resources, availability of magnesium sulphate, urine strip, functional $\mathrm{BP}$ machine and other required logistics. This checklist was adapted from an evaluation survey exploring the quality of antenatal care in government health facilities of Bangladesh. ${ }^{20}$

\section{Data quality assurance and management}

Several data quality checks were in place to correctly record and perform the assigned tasks by the data collectors. The most critical element of the study was the ascertainment of systolic and diastolic BP and protein in urine during reassessment. To measure the $\mathrm{BP}$, manually inflated aneroid sphygmomanometers were used by the study physicians as this instrument was routinely used by the FWVs in UH\&FWCs. This type of sphygmomanometer is more portable and preferable for community-level 
health services than any other BP measuring devices, though considered less accurate than a mercury sphygmomanometer. ${ }^{21} 22$ To minimise the variation in measurements during reassessment, $\mathrm{BP}$ was measured two times for each ANC recipient in the same hand (right arm, unless there was any clinical or physical condition), on a sitting position, and at $5 \mathrm{~min}$ of resting interval between two measurements. Both measurements were recorded during the reassessment by the study physicians and the average of these two measurements was used in the statistical analysis. The standard procedures were also maintained during the 'Non-Communicable Disease Risk Factor Survey Bangladesh 2010,23 and suggested in the 'WHO STEPS guideline'. ${ }^{24}$ For urine reassessment, a sample of urine was collected in a test tube maintaining adequate sterility and hygiene. Reagent strips for urine analysis were used to assess the presence of protein in the sample. The same test-strips were also being used in the selected UH\&FWCs for ANC services as supplied by the government with support from MaMoni HSS.

Study paramedics reviewed each other's ANC observation checklist for completeness before submitting it to the study physician of the team who rechecked each form for missing information or inconsistency. Throughout the study period, one medical officer of the central research team conducted spot-checking that is, made unscheduled visits to study sites, reviewed the collected data on the spot and provided necessary feedback to the study physicians and the paramedics. Regular monitoring and supervision were conducted by the central team of research investigators led by the principal investigator of the study.

A central data management team was responsible for data entry, consistency checking and cleaning. All data were entered into the database using a custom-designed data entry programme with built-in range and consistency checks.

\section{Definition of variables and data analysis}

Four conditions, namely gestational hypertension, preeclampsia, severe pre-eclampsia and eclampsia were collectively considered the hypertensive disorders of pregnancy in this study, and their operational definitions are summarised in table 1 . The diagnostic criteria mentioned in the operational definitions followed the nationally adapted protocol for the detection and management of hypertensive disorders of pregnancy (online supplemental file 1). The study physicians reassessed each ANC recipient, performed the required examinations and applied the diagnostic criteria based on their assessment to reach a diagnosis. For the FWVs, on the other hand, if they made a diagnosis of one of the four hypertensives disorders of pregnancy and recorded it on the ANC register or the ANC card of the recipient, it was considered as the 'case detected by FWV' irrespective of the validity of their assessment for such conditions. In case the FWVs did not record or document any diagnosis for a pregnant woman, the diagnosis was considered as 'no hypertensive disorder of pregnancy'.

Descriptive statistics were used to report the background characteristics of the ANC recipients; competency of the FWVs in screening, detection and management of hypertensive disorders of pregnancy and presented in percentage of cases who received health services (exploring clinical symptoms, performing screening procedures, counselling, providing injectable magnesium sulphate and referral) in adherence to the standard ANC guideline. The background characteristics of the ANC recipients and the findings on screening procedures were

Table 1 Operational definitions of hypertensive disorders of pregnancy

\begin{tabular}{|c|c|}
\hline $\begin{array}{l}\text { Hypertensive } \\
\text { disorders of } \\
\text { pregnancy }\end{array}$ & Diagnostic criteria \\
\hline $\begin{array}{l}\text { Gestational } \\
\text { hypertension }\end{array}$ & $\begin{array}{l}\text { Gestational age: } 20 \text { weeks or more. } \\
\text { Diastolic blood pressure: } 90-109 \mathrm{~mm} \mathrm{Hg} \text {. } \\
\text { Protein in urine: nil. } \\
\text { Absence of all three danger signs - severe headache, blurred vision and upper abdominal pain. }\end{array}$ \\
\hline Pre-eclampsia & $\begin{array}{l}\text { Gestational age: } 20 \text { weeks or more. } \\
\text { Diastolic blood pressure: } 90-109 \mathrm{~mm} \mathrm{Hg} \text {. } \\
\text { Protein in urine: present. } \\
\text { Absence of all three danger signs - severe headache, blurred vision and upper abdominal pain. }\end{array}$ \\
\hline $\begin{array}{l}\text { Severe pre- } \\
\text { eclampsia }\end{array}$ & $\begin{array}{l}\text { Definition\#1: } \\
\text { Gestational age: } 20 \text { weeks or more. } \\
\text { Diastolic blood pressure } 90-109 \mathrm{~mm} \mathrm{Hg} \text {. } \\
\text { Protein in urine: present. } \\
\text { Presence of at least one of the three danger signs-severe headache, blurred vision and upper abdominal pain. } \\
\text { Gestion\#2: } \\
\text { Giastolic blood pressure: } 110 \mathrm{~mm} \text { Hg or more. } \\
\text { Protein in urine: present. }\end{array}$ \\
\hline Eclampsia & $\begin{array}{l}\text { Gestational age: } 20 \text { weeks or more. } \\
\text { Diastolic blood pressure: } 90 \mathrm{~mm} \mathrm{Hg} \text { or more. } \\
\text { Convulsion. }\end{array}$ \\
\hline
\end{tabular}


further stratified by place of service delivery-UH\&FWC and satellite clinics. Pearson's $\chi^{2}$ test was conducted to assess whether participants' characteristics were significantly different between UH\&FWC and satellite clinics and assess the significance of the association between the FWV's practice and place of service delivery.

Inter-rater agreement between FWV and study physician was measured using per cent agreement. Cohen's kappa coefficient along with the $\mathrm{p}$ value was also estimated to account for the possibility of finding the agreement by chance. Cohen's kappa coefficient describes the strength of inter-rater agreement by comparing the observed agreement to the expected agreement, assuming the measurements are independent. ${ }^{25}$ Sensitivity, specificity and positive predictive values (PPVs) were calculated for assessing the validity of FWV's diagnosis of-(i) any hypertensive disorder of pregnancy (including gestational hypertension) and (ii) pre-eclampsia, severe preeclampsia or eclampsia. For sensitivity and specificity analysis of the conditions (i) and (ii), the measurements and diagnosis made by the study physicians were considered as 'true' or 'gold standard'. Sensitivity was expressed as the percentage of 'cases detected by FWV' among those who had 'true' medical criteria for hypertensive disorders of pregnancy, specificity was expressed as the percentage of non-cases detected by FWV among those who did not have 'true' medical criteria for those conditions, and the PPV was expressed as the percentage of 'true' cases among the 'case detected by FWV' as hypertensive disorders of pregnancy. As part of the validity analysis, two scenarios were compared with the study physician's diagnosis (gold-standard) - (i) diagnosis that was made by FWV and recorded in the ANC registers/ ANC card irrespective of using the algorithm present in the national protocol for detecting hypertensive disorders of pregnancy and (ii) post-hoc diagnosis using the information from FWV's measurement of BP and urine assessment, the status of danger signs from study physician's assessment and applying the algorithm during analysis. The latter indicates the extent to which cases could have been accurately diagnosed had the two diagnostic criteria (elevated BP and protein in the urine) been appropriately used as per the national protocol for hypertensive disorders of pregnancy by FWVs.

Pearson's correlation coefficient was reported along with $\mathrm{p}$ values to estimate the relationship between the measurements taken by the FWV and the study physician. The comparison is presented in scatterplot graphs by plotting BP recordings taken by FWVs against those taken by study physicians. Mean and SD of absolute difference of BP readings taken by FWVs and study physicians are calculated to estimate the accuracy of FWV's measurement of diastolic and systolic BP. A first-order polynomial smooth curve of the absolute differences is also fitted in the scatter plot to show the deviation from the 'true' values. Accuracy of urine assessment is presented in the percentage of cases where FWV could correctly identify if there was 'no protein' or 'some protein' in the urine. The results of the urine assessment ascertained by study physicians were considered 'correct'. We used the Strengthening the Reporting of Observational Studies in Epidemiology cross-sectional checklist when writing this manuscript. ${ }^{26}$

\section{Patient and public involvement}

The study objectives and outcome measures were related to assessing the competency of health workers in detecting and managing gestational hypertension, pre-eclampsia, severe pre-eclampsia and eclampsia during ANCs in primary care health facilities in Bangladesh. Pregnant women were not involved in the design of, in the recruitment to and conduct of the study. The results will be disseminated among the health managers and maternal health workers in the government hospitals where the study was conducted.

This study was not a clinical investigation. MaMoni HSS project only provided technical support for the implementation of the nationally adopted intervention of community-based provision of the loading dose of magnesium sulphate to the identified cases of severe preeclampsia and eclampsia through the government health system. Nonetheless, systematic reviews and the efficacy trials of magnesium sulphate have already proven that the drug is safe with no major side effects. ${ }^{27-29}$

\section{RESULT \\ Readiness of UH\&FWCs}

All 26 UH\&FWCs provided antenatal services and the mean $( \pm \mathrm{SD})$ number of ANC provided in a month was 111 $( \pm 83)$ (data not presented in table). All the UH\&FWCs had one designated FWV who provided ANC, childbirth, postnatal care and family planning services. She also conducted regular satellite sessions two times a week to provide the same health services in the satellite clinics. All 26 FWVs received hands-on training on screening, diagnosing and managing hypertensive disorders of pregnancy. In only seven UH\&FWCs (27\%), at least one case of diagnosing and managing pre-eclampsia in the last 3 months preceding the assessment was reported. The BP machines and urine strips for assessing protein in urine were available in all 26 health facilities, but three did not have injectable magnesium sulphate available during the assessment period. The standard national guideline for managing hypertensive disorders during ANC was available in only 16 out of 26 facilities. The readiness of the satellite clinics regarding the service provision and logistics remained similar to that in the UH\&FWC since the FWV carried the ANC logistics with her while attending the satellite sessions.

\section{Characteristics of pregnant women and information on ANC care-seeking}

Of the 1560 pregnant women, $955(61 \%)$ received ANC at UH\&FWCs and the rest at satellite clinics (table 2). About $69 \%$ of women were aged between 20 and 29 years 
Table 2 Characteristics of pregnant women and information on ANC care-seeking

\begin{tabular}{lllll}
\hline $\begin{array}{l}\text { Characteristics, } \\
\mathbf{N}=1560\end{array}$ & $\begin{array}{l}\text { All, } \\
\%\end{array}$ & $\begin{array}{l}\text { UH\&FWC, } \\
\%\end{array}$ & $\begin{array}{l}\text { SC, } \\
\%\end{array}$ & P value $^{*}$ \\
\hline $\begin{array}{l}\text { Total pregnant } \\
\text { women, } \mathrm{n}\end{array}$ & 1560 & 955 & 605 & \\
\hline $\begin{array}{l}\text { Age (in years) } \\
\quad \text { Less than 20 }\end{array}$ & 16.7 & 17.1 & 16.0 & 0.544 \\
20-24 & 38.4 & 38.0 & 39.0 & \\
25-29 & 30.6 & 31.4 & 29.3 & \\
30 and above & 14.4 & 13.5 & 15.7 & \\
Gravida & & & & \\
\hline $\begin{array}{l}\text { Primigravida } \\
\text { Multigravida }\end{array}$ & 38.1 & 38.6 & 41.2 & 0.547 \\
Gestational age & 61.9 & 61.4 & 58.8 & \\
\hline $\begin{array}{l}\text { Less than 20 } \\
\text { weeks }\end{array}$ & 79.9 & 82.0 & 76.7 & 0.011 \\
20 weeks or more & 20.1 & 18.0 & 23.3 & \\
\hline ANC visit† & & & & \\
\hline 1st visit & 59.5 & 54.7 & 67.1 & $<0.0001$ \\
2nd or more & 39.7 & 44.3 & 32.6 & \\
\hline
\end{tabular}

*Pearson's $\chi^{2}$ test.

$\dagger 12$ missing information.

ANC, antenatal check-up; SC, satellite clinic; UH\&FWC, union health and family welfare centre.

and $38 \%$ were first-time mothers (primigravida). One in five women came for ANC after 20 weeks of gestational age; among them, $40 \%$ came for the first antenatal visit. The gestational age and the order of the ANC visits of the participants were significantly different between those who received care at UH\&FWCs and satellite clinics.
Screening and assessment by FWV for hypertensive disorders of pregnancy during ANC

During antenatal service provision, FWVs asked only $8 \%$ of women whether they had a history of hypertension or taking any antihypertensive drug or of pre-eclampsia (if the women had previous pregnancy) (table 3). Symptoms of pre-eclampsia for the current pregnancy such as severe headache, blurring of vision, upper abdominal pain, were assessed among $15 \%, 12 \%$ and $18 \%$ of women, respectively. Only for $12 \%$ of women, any occurrence of convulsion during their current pregnancy was explored. FWVs were more likely to ask these screening questions if the services were given at a UH\&FWC than in a satellite clinic $(\mathrm{p}<0.05)$. BP and urine assessment, two critical examinations for diagnosing the presence and severity of hypertensive disorders of pregnancy, were conducted among $97 \%$ and $76 \%$ of the women, respectively.

\section{Accuracy of FWV's assessment of BP and urine BP measurement}

Figures 1 and 2 plot the diastolic and systolic BPs of pregnant women, respectively, as recorded by the FWVs against those recorded by the study physicians. Both figures show a tendency of FWVs to round off the BPs to the nearest tenth of the unit which either underestimated or over-estimated the 'true' values as evident by comparing with the study physicians' measurements. The correlation coefficients of the measurements taken by FWV and the study physician were 0.50 ( $\mathrm{p}$ value $<0.0001$ ) for diastolic pressure and 0.60 ( $p$ value $<0.0001$ ) for systolic pressure, which indicates a significant moderate positive relationship. Overall, the mean $( \pm \mathrm{SD})$ absolute difference between FWV and the study physician's measurement of diastolic and systolic BP of the same person was $7.1 \pm 7.6$ and $7.5 \pm 8.4 \mathrm{~mm} \mathrm{Hg}$, respectively (not

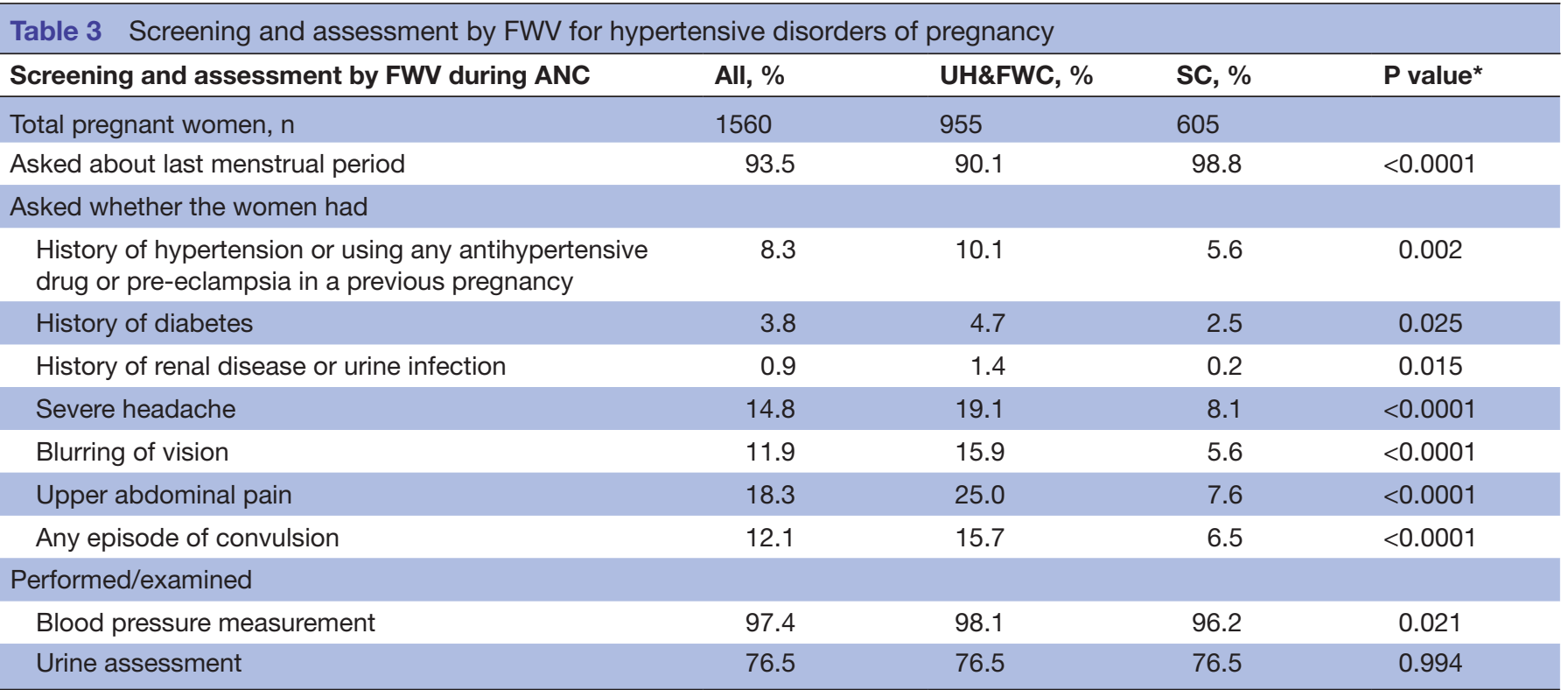

*Pearson's $\chi^{2}$ test.

ANC, antenatal check-up; FWV, Family Welfare Visitor; SC, satellite clinics; UH\&FWC, union health and family welfare centre. 


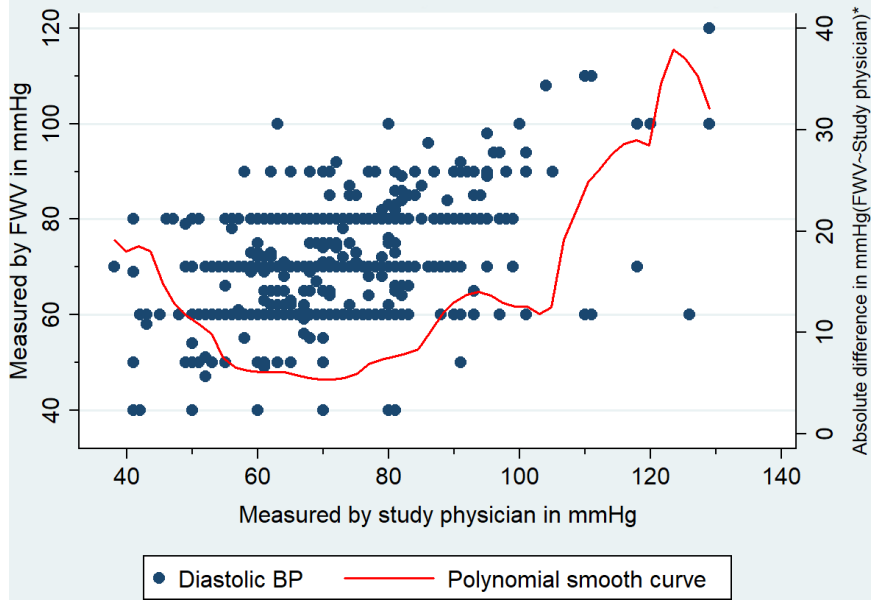

Figure 1 Diastolic BP: recorded by FWV versus study physician. BP, blood pressure; FWV, Family Welfare Visitor.

presented in the table). The polynomial smooth curve of the absolute differences between the study physician and FWV's measurement shows larger differences $(>10 \mathrm{~mm}$ $\mathrm{Hg}$ ) when the 'true' diastolic pressure was higher than $100 \mathrm{~mm} \mathrm{Hg}$ or 'true' systolic pressure was higher than $160 \mathrm{~mm} \mathrm{Hg}$. The difference also widened when 'true' diastolic pressure was below $50 \mathrm{~mm} \mathrm{Hg}$ or 'true' systolic pressure was below $100 \mathrm{~mm} \mathrm{Hg}$.

\section{Urine assessment}

Although there was high correct identification of 'no protein' in urine, only $27 \%$ of those who had 'some protein' were accurately diagnosed by FWVs (figure 3).

\section{Status of hypertensive disorders of pregnancy}

The 'true' prevalence for gestational hypertension, preeclampsia and severe pre-eclampsia diagnosed by the study physicians, was $2.1 \%, 1.9 \%$ and $1.0 \%$, respectively (table 4). The numbers of cases identified by the FWVs were substantially lower than the 'true' prevalence for all three types of hypertensive disorders. No woman was

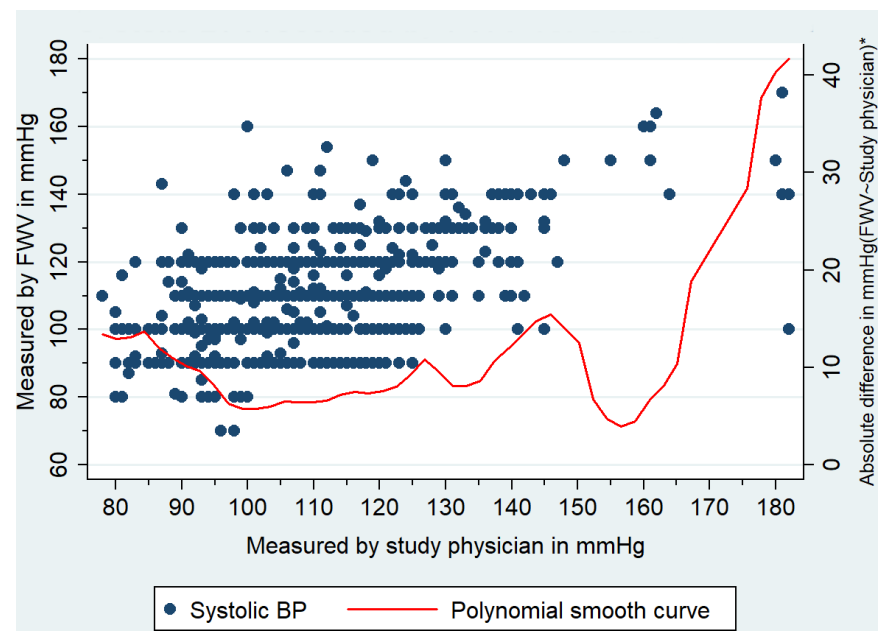

Figure 2 Systolic BP: recorded by FWV versus study physician. BP, blood pressure; FMV, Family Welfare Visitor.
$\%$ of pregnant women correctly identified by FWV for protein in urine, $\mathrm{N}=1194$

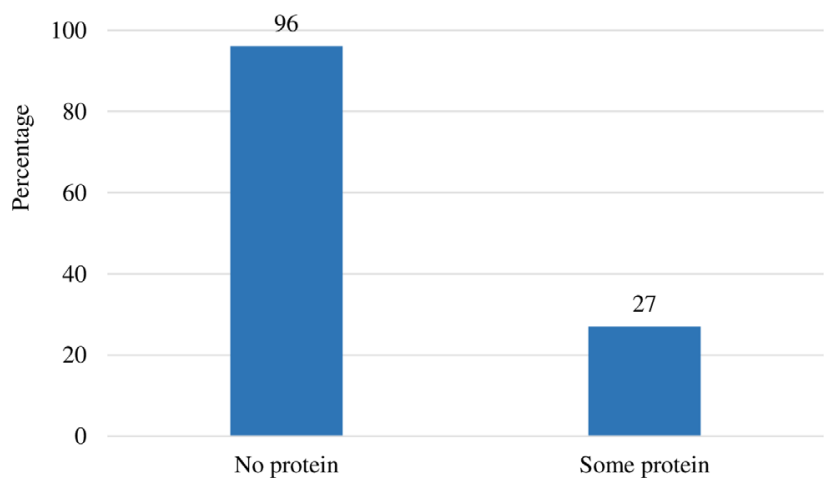

Figure 3 Protein in urine: accurately diagnosed by FWV. FMV, Family Welfare Visitor.

diagnosed with eclampsia by the study physician or FWV. Although the numbers are too small to compare, the FWVs could detect a higher proportion of cases of hypertensive disorders if they provided care in UH\&FWCs than in satellite clinics.

\section{Validity of the diagnosis of hypertensive disorders of pregnancy}

FWV's diagnosis recorded in ANC register

Only in 3\%, $7 \%$ and $25 \%$ of true cases of gestational hypertension, pre-eclampsia and severe pre-eclampsia, respectively, FWVs made a correct diagnosis during ANC (table 5). Per cent agreement for the diagnosed cases of hypertensive disorders of pregnancy is $9 \%$ and kappa statistics is 0.05 ( $p$ value 0.0125 ). For identification of any hypertensive disorders, sensitivity and PPV is only $14 \%$ and $50 \%$, respectively. For identification of pre-eclampsia or severe pre-eclampsia or eclampsia, sensitivity and PPV are $18 \%$ and $80 \%$, respectively. In both cases, specificity is high $(>99 \%)$.

\section{Post-hoc diagnosis based on FWV's assessment of BP and urine} When the diagnosis was made by applying the algorithm present in national protocol based on the two diagnostic criteria (elevated $\mathrm{BP}$ and protein in the urine) measured by FWVs and status of three danger signs assessed by study physicians the accuracy of diagnosis (ie, the percentage of 'true' cases accurately diagnosed), was markedly increased from that of the FWVs' original diagnosis of the conditions (table 5). For instance, at least $63 \%$ of 'true' cases of severe pre-eclampsia could be diagnosed if the protocol had been applied correctly by an FWV based on her assessment of BP and urine. The per cent agreement also improves from $9 \%$ to $29 \%$ and kappa statistics is 0.19 ( $\mathrm{p}$ value $<0.0001$ ). Moreover, in this scenario, both sensitivity and PPV of diagnosing 'any hypertensive disorder' and 'pre-eclampsia and/or severe pre-eclampsia' are markedly increased. 
Table 4 Prevalence of hypertensive disorders of pregnancy

\begin{tabular}{|c|c|c|c|c|c|c|}
\hline \multirow{3}{*}{$\begin{array}{l}\text { Hypertensive disorders } \\
\text { of pregnancy }\end{array}$} & \multicolumn{6}{|c|}{ Cases identified/diagnosed by } \\
\hline & \multicolumn{3}{|c|}{ Study physician* } & \multicolumn{3}{|c|}{ FWV† } \\
\hline & All, n (\%) & UH\&FWC, n (\%) & SC, n (\%) & All, $n$ (\%) & UH\&FWC, n (\%) & SC, n (\%) \\
\hline Gestational hypertension & $32(2.1)$ & $18(1.9)$ & $14(2.3)$ & $12(0.8)$ & $11(1.1)$ & $1(0.1)$ \\
\hline Pre-eclampsia & $29(1.9)$ & $18(1.9)$ & $11(1.8)$ & $6(0.4)$ & $4(0.4)$ & $2(0.3)$ \\
\hline
\end{tabular}

*According to the national protocol for hypertensive disorders of pregnancy based on the information recorded by study physician after reassessment of the pregnant women.

†According to FWV's note recorded in antenatal check-up (ANC) register/ANC card after ANC was provided.

FWV, Family Welfare Visitor; SC, satellite clinics; UH\&FWC, union health and family welfare centre.

\section{Management provided by FWV}

The women who were diagnosed to have any hypertensive disorders of pregnancy by FWVs were more likely to be counselled on at least one danger sign of pre-eclampsia (severe headache, blurring of vision and upper abdominal pain) than those without such diagnosis $(41 \%$ vs $19 \%$, p value 0.008 ). The FWVs provided antihypertensive drugs only for those with the diagnosis of gestational hypertension and pre-eclampsia (table 6). All four cases of severe pre-eclampsia were given a loading dose of intramuscular magnesium sulphate and three among them were referred to a higher facility.

\section{DISCUSSION}

Our study identified gaps in diagnosing hypertensive disorders of pregnancy during ANC in communitylevel satellite sessions and at primary healthcare centres in three districts of Bangladesh where USAID funded MaMoni HSS project intervened to improve maternal healthcare services. The undiagnosed or misdiagnosed cases could be linked with inadequate and inaccurate screening procedures performed by the health workers called FWVs. The study also observed inadequate history taking, inaccuracy in BP measurement and/or recording, and urine assessment for protein. A small proportion of the women who required antihypertensive drugs or counselling on disease management received such. All the detected cases of severe pre-eclampsia received the first dose of injectable magnesium sulphate as per the protocol.

The 'focused ANC package' by the WHO emphasised BP measurement and urine assessment during all ANC visit irrespective of gestational age to screen hypertensive disorders of pregnancy. ${ }^{19}{ }^{30}$ In our study, nearly all women had their BP measured and about three-fourths had their urinary protein assessed. The MaMoni HSS project trained FWVs on diagnosis and management of pre-eclampsia and eclampsia before the study and the training could have influenced the high coverage of the screening procedures performed by the FWVs. Detection of hypertensive disorders of pregnancy, however, requires screening for maternal comorbidities and proper history taking for danger signs of pre-eclampsia and convulsion during the current pregnancy, which was observed inadequate in our study. Nevertheless, the poor quality of ANC is consistent with that in other low/middle-income (LMIC) countries including Bangladesh. ${ }^{2031}$ The practice was also significantly lower when the women received ANC in satellite or outreach sessions compared with in-facility antenatal services as found in another study conducted in rural Bangladesh. ${ }^{32}$ The satellite or outreach sessions are often hosted at the household of local rural elites on their acceptance and the health workers providing ANC in UH\&FWCs need to carry all the necessary equipment and register books from the UH\&FWC to the place of satellite sessions on the designated day of the week. ${ }^{14}$ Due to the makeshift nature of the satellite clinics, access to clean toilet facilities and provision of adequate privacy during physical examinations are often difficult to ensure at the service delivery points. ${ }^{33}$ Despite having the required equipment for $\mathrm{ANC}$, poorer readiness of satellite clinics might result in poorer performance of some critical components of ANC such as assessments of dangers signs, BP measurement and urine assessment at those clinics than in the health facility. ${ }^{203}$

Initiatives to improve quality of care often focus on bridging the coverage gap of specific activities by the health workers rather than focusing on the validity of the performed activities or developing reliable screening tools. ${ }^{31}{ }^{34}$ For instance, BP measurement and urine assessment are two key diagnostic criteria for hypertensive disorders of pregnancy, yet their accurate ascertainment receives low priority in the training schemes for health workers in LMICs. ${ }^{35}$ Our study found inconsistency in measured BP between the health workers' assessment and the 'true' value especially when the BP was beyond the normal range. This might allude to the tendency of the FWVs to record BP within the normal range without accurately measuring the $\mathrm{BP}$ of the ANC client. The study also detected terminal digit bias for both systolic and diastolic BP recordings, that is, the tendency to report the values ending in zero. One study suggests that the occurrence of recordings ending in zero 


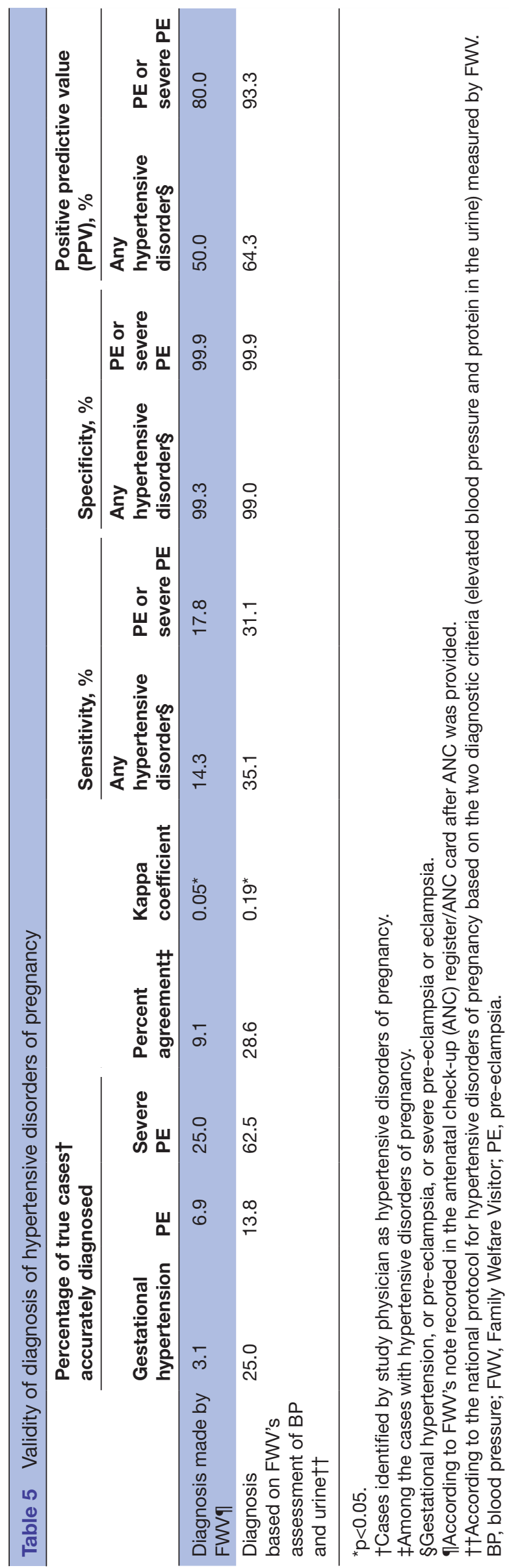

in more than $30 \%$ cases, in general, indicates imprecise and inaccurate recording. ${ }^{36}$ Periodic checking of the ANC registers for terminal digit bias by the supervisors could minimise such practice.

Despite the overall low sensitivity and PPVs for detecting hypertensive disorders of pregnancy by the FWVs, our study found an increase in these parameters when the algorithm present in the pre-eclampsia protocol was applied to diagnose the disease condition using the recordings of the screening tests performed by FWVs. This indicates either poor adherence to the evidence-based guideline or improper interpretation of the national protocol by the FWVs while reaching a diagnosis. Unavailability of the ANC protocol in 16 out of the 26 assessed health facilities could further worsen the knowledge gap of the health workers regarding the disease diagnostic criteria. A landscapeanalysis on pre-eclampsia and eclampsia conducted in Bangladesh concluded that pre-eclampsia/eclampsia guideline was not available in any of the assessed community-level primary healthcare centres, that is, UH\&FWCs and only $8 \%$ of health workers working there knew that a national guideline on diagnosis and management of pre-eclampsia/ eclampsia existed. ${ }^{15}$ Although we have not assessed the reasons for the unavailability of the ANC protocol, lack of regular monitoring of the presence and use of guidelines in community level health facilities and lack of knowledge of the health workers about the content of the guidelines are some critical barriers to maintaining and keeping the guidelines in the facility. ${ }^{185}{ }^{37}$ In addition to putting effort from the central level to make the guidelines available in health facilities, the ANC providers should be made aware of the diagnostic criteria of hypertensive disorders of pregnancy and trained to accurately assess danger signs, measure $\mathrm{BP}$ and urinary protein. A review paper on improving the quality of care in LMICs suggested that capacity development training should also maintain strong supportive supervision, along with the provision of on-site feedback based on health workers' performances, and audit of ANC registers and case reports to improve health workers' adherence to the evidence-based guidelines. ${ }^{38}$

In our study, a subtotal of 16 'true' cases of severe preeclampsia was identified by the study physicians and these cases required the first dose of magnesium sulphate injection before their referral to a higher-level facility. Since only four of them were correctly identified by the FWVs, the rest received no magnesium sulphate injection due to the missed diagnosis of the cases. The study physicians, however, had sent these women back to the FWV for re-evalutaion and guaranteed that they received appropriate care on ethical grounds. Counselling regarding the hypertensive diseases of pregnancy or the provision of antihypertensive drugs to detected cases was also inadequate. The failure in the timely management of hypertensive disorders of pregnancy, therefore, poses a serious challenge in preventing maternal morbidity and mortality in LMICs. Besides improving the knowledge and skill of the ANC providers, prediction of risk and detection of cases can be aided by initiating a computerised clinical decision support system (CDSS) 
Table 6 Management of hypertensive disorders identified by FWV during antenatal check-up at union health and family welfare centre

\begin{tabular}{|c|c|c|c|c|}
\hline \multirow[b]{2}{*}{ Cases diagnosed by FWV } & \multicolumn{3}{|c|}{ Received in-facility management, $n(\%)$} & \multirow[b]{2}{*}{ Referred, n (\%) } \\
\hline & Counselling* & Antihypertensive† & Magnesium sulphate‡ & \\
\hline No hypertensive disorders, $n=1538$ & $287(18.7)$ & $\mathrm{n} / \mathrm{a}$ & $\mathrm{n} / \mathrm{a}$ & $\mathrm{n} / \mathrm{a}$ \\
\hline Pre-eclampsia, $\mathrm{n}=6$ & 2 (33.3) & $1(16.7)$ & $\mathrm{n} / \mathrm{a}$ & $3(50.0)$ \\
\hline Severe pre-eclampsia, $n=4$ & $3(75.0)$ & $0(0)$ & $4(100.0)$ & $3(75.0)$ \\
\hline
\end{tabular}

${ }^{*}$ Counselling on at least one danger signs of pre-eclampsia.

†Oral hydralazine, nifedipine, labetalol or methyldopa.

$\ddagger$ A loading dose of intramuscular magnesium sulphate $(10 \mathrm{gm})$.

FWV, Family Welfare Visitor.

which has been proven effective for improved providercare seeker communication and clinical decision making. ${ }^{39}$ Unlike in developed countries, where CDSS was effective in improving health workers' performances ${ }^{40}$ implementation of such technology in LMICs could be challenging in the context of poor availability of health information, inadequate infrastructure and limitation of skilled human resources. ${ }^{41}$ Since the adoption of the national eHealth policy in 2011, ${ }^{42}$ Bangladesh is progressing with establishing electronic management information system (e-MIS) in the health facilities and has started to provide community health workers with computer tablets or smartphones to deliver essential health services on the pilot basis. ${ }^{43}$ The integration of CDSS into the e-MIS initiative could contribute to the sustainability of improving the quality of care during ANC, and thus the screening of hypertensive disorders. Further research is needed to identify and address potential constraints for smooth implementation of the decision support technology to ensure proper detection and management of gestational hypertensive disorders in Bangladesh and other LMICs.

\section{CONCLUSION}

Consistent application of the national protocol supports clinical-decision making by health workers during the provision of ANC. Efforts should be directed at accurate application and interpretation of the national protocols or guidelines by health workers as a prerequisite for task shifting for the detection and management of hypertensive disorders of pregnancy at primary level health facilities and outreach centres. The training of ANC health workers should emphasise the risk assessment of pregnant women with a practical demonstration on assessing the diagnostic criteria and management of eclampsia and pre-eclampsia. Strengthening the monitoring and supervision can further improve the screening of pre-eclampsia and severe preeclampsia and timely management of such cases to prevent eclampsia.

\section{Author affiliations}

${ }^{1}$ Maternal and Child Health Division, International Centre for Diarrhoeal Disease Research, Dhaka, Bangladesh
${ }^{2}$ Faculty of Medicine and Health, Sydney School of Public Health, The University of Sydney, Sydney, New South Wales, Australia

${ }^{3}$ Health Promotion, Education and Behavior, Arnold School of Public Health, University of South Carolina, Columbia, South Carolina, USA

${ }^{4}$ Shukhi Jibon, Pathfinder International, Dhaka, Bangladesh

${ }^{5}$ Urban Health Initiative, JHPIEGO, Kabul, Afghanistan

${ }^{6}$ Maternal Health \& Family Planning, JHPIEGO, Dhaka, Bangladesh

${ }^{7}$ MaMoni Health Systems Strengthening, Save the Children Bangladesh, Dhaka, Bangladesh

Acknowledgements We acknowledge the Director (MCH Services) of Directorate General of Family Planning and Deputy Director Family Planning of Habiganj, Noakhali and Lakhsmipur districts for allowing us to conduct the study. We also acknowledge the support from health workers and pregnant women for accepting us to observe the service delivery procedure and providing necessary information. Also, icddr,b gratefully acknowledges the following donors who provide unrestricted support: Government of the People's Republic of Bangladesh; Global Affairs Canada (GAC); Swedish International Development Cooperation Agency (Sida) and the Department for International Development, (UKAid). We used the STROBE crosssectional checklist when writing this manuscript.

Contributors SMB, SEA and JG conceptualised the paper. SMB, ANSK, SMR, NLH, MAK, SSP and IIM were involved in data acquisition, analysis, interpretation and literature review. SMB, ANSK and SMR prepared the first draft. SMB, ANSK, SMR, NLH, MAK, IIM, SR, SEA and JG contributed to the revision and preparation of the final draft. All authors have reviewed and approved the final manuscript.

Funding This study was undertaken, and the article was produced as a part of MaMoni Health Systems Strengthening's (MaMoni HSS) learning agenda with the generous support of the American people through the US Agency for International Development (USAID); Associate Cooperative Agreement No. AID-338-LA-13-00004. The views expressed in this article are solely the views of the authors and do not reflect the views of the US Agency for International Development or the US Government.

Competing interests None declared.

Patient consent for publication Not required.

Ethics approval The study protocol was reviewed and approved by the Ethical Review Committee (ERC) of the International Center for Diarrheal Disease Research, Bangladesh (icddr,b) (protocol number PR-17084). Permission was taken from the Ministry of Health and Family Welfare and health managers of the selected health facilities were informed about the study. Written informed consent was obtained from the FWVs and verbal consent was sought from ANC service recipients during clinical observations. The respondents participated in the study voluntarily and had the right to withdraw themselves from the study at any time even after giving the consent. Privacy, anonymity and confidentiality of the data were strictly practised and restrictions on access to data were enforced.

Provenance and peer review Not commissioned; externally peer reviewed.

Data availability statement Data are available in a public, open access repository. Technical appendix, statistical code and dataset available from a public repository (https://doi.org/10.6084/m9.figshare.12554330.v1). 
Supplemental material This content has been supplied by the author(s). It has not been vetted by BMJ Publishing Group Limited (BMJ) and may not have been peer-reviewed. Any opinions or recommendations discussed are solely those of the author(s) and are not endorsed by BMJ. BMJ disclaims all liability and responsibility arising from any reliance placed on the content. Where the content includes any translated material, BMJ does not warrant the accuracy and reliability of the translations (including but not limited to local regulations, clinical guidelines, terminology, drug names and drug dosages), and is not responsible for any error and/or omissions arising from translation and adaptation or otherwise.

Open access This is an open access article distributed in accordance with the Creative Commons Attribution Non Commercial (CC BY-NC 4.0) license, which permits others to distribute, remix, adapt, build upon this work non-commercially, and license their derivative works on different terms, provided the original work is properly cited, appropriate credit is given, any changes made indicated, and the use is non-commercial. See: http://creativecommons.org/licenses/by-nc/4.0/.

\section{ORCID iD}

Abdullah Nurus Salam Khan http://orcid.org/0000-0002-8694-4349

\section{REFERENCES}

1 Hutcheon JA, Lisonkova S, Joseph KS. Epidemiology of preeclampsia and the other hypertensive disorders of pregnancy. Best Pract Res Clin Obstet Gynaecol 2011;25:391-403.

2 Wallis AB, Saftlas AF, Hsia J, et al. Secular trends in the rates of preeclampsia, eclampsia, and gestational hypertension, United States, 1987-2004. Am J Hypertens 2008;21:521-6.

3 Ananth CV, Savitz DA, Williams MA. Placental abruption and its association with hypertension and prolonged rupture of membranes: a methodologic review and meta-analysis. Obstet Gynecol 1996;88:309-18.

4 Kassebaum NJ, Bertozzi-Villa A, Coggeshall MS, et al. Global, regional, and national levels and causes of maternal mortality during 1990-2013: a systematic analysis for the global burden of disease study 2013. Lancet 2014;384:980-1004.

5 Duley L. editor The global impact of pre-eclampsia and eclampsia. In: Seminars in Perinatology. Elsevier, 2009.

6 National Institute of Population Research and Training (NIPORT). Bangladesh maternal mortality survey 2010. Dhaka, Bangladesh: NIPORT, MEASURE Evaluation, icddr,b, 2012.

7 Begum MR, Begum A, Quadir E, et al. Eclampsia: still a problem in Bangladesh. MedGenMed 2004;6:52.

8 Villar J, Carroli G, Wojdyla D, et al. Preeclampsia, gestational hypertension and intrauterine growth restriction, related or independent conditions? Am J Obstet Gynecol 2006;194:921-31.

9 Pettit F, Brown MA. The management of pre-eclampsia: what we think we know. Eur J Obstet Gynecol Reprod Biol 2012;160:6-12.

10 Sheth SS, Chalmers I. Magnesium for preventing and treating eclampsia: time for international action. Lancet 2002;359:1872-3.

11 Souza JP, Gülmezoglu AM, Vogel J, et al. Moving beyond essential interventions for reduction of maternal mortality (the who multicountry survey on maternal and newborn health): a crosssectional study. Lancet 2013;381:1747-55.

12 Rawlins B, Plotkin M, Rakotovao JP, et al. Screening and management of pre-eclampsia and eclampsia in antenatal and labor and delivery services: findings from cross-sectional observation studies in six sub-Saharan African countries. BMC Pregnancy Childbirth 2018;18:346.

13 Ansari N, Manalai P, Maruf F, et al. Quality of care in early detection and management of pre-eclampsia/eclampsia in health facilities in Afghanistan. BMC Pregnancy Childbirth 2019;19:36.

14 Ministry of Health and Family Welfare (MOHFW). Bangladesh health Bulletin 2017. Bangladesh: Management Information System: Directorate General of Health Services, 2018.

15 Warren C, Hossain S, Nur RA, et al. Landscape analysis on preeclampsia and eclampsia in Bangladesh. Washington, DC: Population Council, 2015.

16 Khan ANS, Billah SM, Mannan I, et al. A cross-sectional study of partograph utilization as a decision making tool for referral of abnormal labour in primary health care facilities of Bangladesh. PLOS One 2018;13:e0203617.

17 World Health Organization. Who antenatal care randomized trial: manual for the implementation of the new model. Geneva: World Health Organization, 2002.
18 Williams A, Khan MA, Moniruzzaman M, et al. Management of preeclampsia, severe preeclampsia, and eclampsia at primary care facilities in Bangladesh. Glob Health Sci Pract 2019;7:457-468.

19 Lawn J, Kerber K. Opportunities for Africas newborns: practical data policy and programmatic support for newborn care in Africa, 2006.

20 Billah SM, Saha KK, Khan ANS, et al. Quality of nutrition services in primary health care facilities: implications for integrating nutrition into the health system in Bangladesh. PLoS One 2017;12:e0178121.

21 Ngene NC, Moodley J. Blood pressure measurement in pregnancy and in hypertensive disorders of pregnancy: devices, techniques and challenges. Cardiovasc J Afr 2019;30:120-9.

22 Shennan AH, De Greeff A. Measuring blood pressure in pregnancy and pre-eclampsia. In: Pre-eclampsia, etiology and clinical practice. , 2007: 18, 258-75.

23 Zaman MM, Rahman MM, Rahman MR, et al. Prevalence of risk factors for non-communicable diseases in Bangladesh: results from steps survey 2010. Indian J Public Health 2016;60:17-25.

24 Bonita R, Winkelmann R, Douglas KA. The WHO Stepwise approach to surveillance (STEPS) of non-communicable disease risk factors. In: Global behavioral risk factor surveillance. Springer, 2003: 9-22.

25 McHugh ML. Interrater reliability: the kappa statistic. Biochem Med 2012;22:276-82.

26 von Elm E, Altman DG, Egger M, et al. The strengthening the reporting of observational studies in epidemiology (STROBE) statement: guidelines for reporting observational studies. Ann Intern Med 2007;147:573-7.

27 Duley L, Gülmezoglu AM, Henderson-Smart DJ, et al. Magnesium sulphate and other anticonvulsants for women with pre-eclampsia. Cochrane Database Syst Rev 2010;49.

28 Witlin AG, Sibai BM. Magnesium sulfate therapy in preeclampsia and eclampsia. Obstet Gynecol 1998;92:883-9.

29 Coetzee EJ, Dommisse J, Anthony J. A randomised controlled trial of intravenous magnesium sulphate versus placebo in the management of women with severe pre-eclampsia. BJOG 1998;105:300-3.

30 Ahmed S, Hill K. Maternal mortality estimation at the subnational level: a model-based method with an application to Bangladesh. Bull World Health Organ 2011;89:12-21.

31 Hodgins S, D'Agostino A. The quality-coverage gap in antenatal care: toward better measurement of effective coverage. Glob Health Sci Pract 2014;2:173-81.

32 Jo Y, Alland K, Ali H, et al. Antenatal care in rural Bangladesh: current state of costs, content and recommendations for effective service delivery. BMC Health Serv Res 2019;19:1-13.

33 Ministry of Health and Family Welfare (MOHFW). Independent evaluation of community-based health services in Bangladesh. Ministry of Health and Family Welfare, Directorate General of Health Services, Community Based Health Care programme and World Health Organization (WHO) Bangladesh, 2019.

34 Tunçalp Ö, Were WM, MacLennan C, et al. Quality of care for pregnant women and newborns-the who vision. BJOG 2015;122:1045-9.

35 Firoz T, Sanghvi $\mathrm{H}$, Merialdi M, et al. Pre-eclampsia in low and middle income countries. Best Pract Res Clin Obstet Gynaecol 2011;25:537-48.

36 Grim CM, Grim CE. Manual Blood Pressure Measurement-Still the Gold Standard. In: Hypertension medicine. Springer, 2001: 131-45.

37 Theodorou M, Stafylas P, Kourlaba G, et al. Physicians' perceptions and adherence to guidelines for the management of hypertension: a national, multicentre, prospective study. Int $J$ Hypertens 2012;2012:1-11.

38 Rowe AK, de Savigny D, Lanata CF, et al. How can we achieve and maintain high-quality performance of health workers in low-resource settings? Lancet 2005;366:1026-35.

39 Roshanov PS, Misra S, Gerstein HC, et al. Computerized clinical decision support systems for chronic disease management: a decision-maker-researcher partnership systematic review. Implement Sci 2011;6:92.

40 Garg AX, Adhikari NKJ, McDonald H, et al. Effects of computerized clinical decision support systems on practitioner performance and patient outcomes: a systematic review. JAMA 2005;293:1223-38.

41 Bediang G, Bagayoko CO, Geissbuhler A. Medical decision support systems in Africa. Yearb Med Inform 2010;19:47-54.

42 Bhuiyan SH. Modernizing Bangladesh public administration through e-governance: benefits and challenges. Gov Inf Q 2011;28:54-65.

43 Walker D, Azim T. Bangladesh's electronic management information system: using digital technology to link community data with facility data: case study Chapel Hill. NC: MEASURE Evaluation, 2018. https://wwwmeasureevaluationorg/resources/publications/tr-18-270 